Institutions

Markets

Technologies

IMT

INSTITUTE

FOR ADVANCED

STUDIES

LUCCA
\#02

2015

IMT LUCCA EIC WORKING

PAPER SERIES 02

May 2015

\title{
Longevity assets and pre-retirement consumption/portfolio decisions
}

Francesco Menoncin Luca Regis 
ISSN 2279-6894

IMT LUCCA EIC WORKING PAPER SERIES \#02/2015

(C) IMT Institute for Advanced Studies Lucca

Piazza San Ponziano 6, 55100 Lucca

\section{Longevity assets and pre- retirement consumption/portfolio decisions}

\section{Francesco Menoncin}

University of Brescia

\section{Luca Regis}

IMT Institute for Advanced Studies Lucca 


\title{
Longevity assets and pre-retirement consumption/portfolio decisions
}

\author{
Francesco Menoncin ${ }^{\mathrm{a}}$, Luca Regis ${ }^{\mathrm{b}}$ \\ ${ }^{a}$ University of Brescia - Department of Economics and Business, Via S. Faustino 74/B, 25122 \\ Brescia, Italy. Tel: 0039-030-2988806. Fax: 0039-030-2988837. E-mail: \\ francesco.menoncin@unibs.it \\ ${ }^{b} I M T$ Institute for Advanced Studies - AXES Research Unit, Piazza San Francesco 19, 55100 Lucca, \\ Italy.Tel.0039-0583-432654\%.E-mail: luca.regis@imtlucca.it
}

\begin{abstract}
We derive a closed form solution for the optimal consumption/investment problem of an agent whose force of mortality is stochastic and whose financial horizon coincides with a fixed retirement date. The investment set includes a longevity asset, as a derivative on the force of mortality. We explore the optimal choices of a representative agent having Hyperbolic Absolute Risk Aversion preferences on both consumption and final wealth. Our numerical analysis shows that individuals optimally invest a large fraction of their wealth in the longevity asset. In our base scenario, calibrated on real world data, a 60-year old male retiring after 5 years should invest around $88 \%$ of his wealth in the longevity asset. Such a percentage decreases as time to retirement decreases. We explore sensitivity of our results to market and individual characteristics.

Keywords: longevity risk, pre-retirement savings, consumption/portfolio choices, HARA preferences.

JEL classification: C61, G11.
\end{abstract}




\section{Introduction}

Despite the relevant and increasing hedging need of pension funds and annuity providers, the market for longevity risk, i.e. the risk of unexpected changes in the mortality of a group of individuals, is not sufficiently liquid yet.

Many reasons may have contributed to undermine a rapid development of the market, such as the lack of standardisation, informational asymmetries, and basis risk. Nevertheless, recent developments provide a sound technology for modelling the systematic randomness in mortality (see e.g. Lee and Carter, 1992), for designing and evaluating hedging instruments (Blake et al., 2006 and Denuit et al., 2007) and for managing longevity risk (Barrieu et al., 2012).

Furthermore, the transfer of longevity risk from pension funds to re-insurers has become more and more common, although on an over-the-counter basis. For instance, the volume of outstanding UK longevity swaps has reached 50 billion pounds as of the end of 2014, with a prevalence of very large deals, such as the 16 billion pounds swap between BT Pension Scheme and Prudential and the 12 billion euros Delta Lloyd/RGA Re index-based transaction. Investment banks have been also actively in the transactions. Between 2008 and 2014, alongside reinsurance specialists, JP Morgan, Credit Suisse, Goldman Sachs, Deutsche Bank and Société Générale were involved in longevity deals (Luciano and Regis, 2014).

Longevity-linked products should be of interest to asset managers for at least two reasons: their low correlation to other asset classes (at least in the short run, see Loeys et al., 2007), and their effectiveness in hedging individual investors against the unexpected fluctuations of their subjective discount factors, which take into account 
lifetime uncertainty (Yaari, 1965, Merton, 1971, Huang et al., 2012).

The aim of this paper is to analyse the optimal consumption and portfolio choices of an investor subject to longevity risk prior to retirement. The agent can invest in a friction-less, arbitrage free, and complete financial market where both traditional assets (bonds and stocks) and a longevity bond are listed.

Contrary to life-cycle analysis, we focus on pre-retirement decisions. This choice allows us to reproduce the point of view of asset managers or pension funds whose investment horizon coincides with the time of annuitisation of individual's wealth. We thus consider a fixed deterministic retirement age (in contrast with Farhi and Panageas, 2007 and Dybvig and Liu, 2010 for instance, who consider an endogenous retirement choice), which coincides with the time horizon of the investor.

An extensive literature has explored consumption and investment decisions when mortality contingent claims are present. In particular, Huang and Milevsky (2008) analyse the decisions of families in the presence of income risk and life insurance. Explicit solutions are also obtained by Pirvu and Zhang (2012) with stochastic asset prices drifts and inflation risk and by Kwak and Lim (2014) with constant relative risk aversion (CRRA) preferences. All these papers consider a deterministic force of mortality, while we model it as a stochastic process. We describe longevity risk by means of a doubly stochastic process whose intensity follows a continuous-time diffusion (as in Dahl, 2004). This process may be correlated with the other state variables. With stochastic mortality, both individuals and annuity/life insurance sellers are exposed to unexpected changes in the force of mortality, implying under or over reserving.

The optimal investment problem of pension funds in the accumulation phase has 
been studied extensively, for instance by Battocchio et al. (2007) and Delong et al. (2008). In this paper, we focus instead on the effects of longevity risk and its hedging on individual's consumption and portfolio decisions. The role of longevity-linked assets in investor's optimal portfolio has been addressed first by Menoncin (2008). Maurer et al. (2013), solving a life-cycle portfolio investment problem with longevity risk, assess the importance of variable annuities to smooth consumption, while Horneff et al. (2010) analyse the role of deferred annuities. They find that these products should optimally account for $78 \%$ of the financial wealth of a retiree.

While insurance products are non-marketable, longevity assets on the market allow individuals to dynamically hedge against mortality fluctuations (we abstract from transaction costs). Cocco and Gomes (2012) analyse, in the context of a life-cycle model, the demand for a perfect hedge against shocks in the life expectancy of a CRRA agent. They study the optimal investment in a longevity bond, which is akin to our zero-coupon longevity asset. In their numerical simulations, they find that individuals - at old ages and especially approaching retirement - should invest a relevant fraction of their wealth in the longevity asset.

Our main contribution consists in providing a closed form solution to the (finitehorizon) problem of an agent prior to retirement, endowed with a general Hyperbolic Absolute Risk Aversion (HARA) class of preferences when mortality intensity is stochastic. We also provide a calibrated application, which allows to appreciate the relevance of longevity products in the optimal agent's portfolio.

Under reasonable stochastic models and calibration for both mortality intensity and interest rate, we find that individuals should optimally invest a relevant fraction of their 
wealth in a longevity bond. A 60-year old US male, who wants to retire at age 65 should optimally invest around $88 \%$ of his portfolio in the (zero market price of risk) longevity bond and then progressively decrease this share approaching retirement. We explore the sensitivity of our results to both individual and market characteristics, finding that the optimal demand for longevity bonds: (i) is higher for 60-year old US females than for 60-year old US males; (ii) reduces (but very slightly) when the agent displays a more conservative behaviour (either high risk aversion, or high minimum consumption or high final wealth minimum level); (iii) remains positive over the whole horizon unless the risk premium is unreasonably low. These last two results are robust for younger agents.

The outline of the paper is the following. Section 2 describes the model setup, while Section 3 describes the individual preferences and the maximisation problem. The optimal consumption and portfolio are found in closed form. Section 4 provides a calibrated application based on US data. Finally, Section 5 concludes, and some technical derivations are left to two appendices.

\section{The model setup}

\subsection{State variables}

On a continuously open and friction-less financial market over the time set $\left[t_{0},+\infty[\right.$, the economic framework is described by a set of $s$ state variables $z(t) \in \mathbb{R}^{s}$ which solve the following (matrix) stochastic differential equation:

$$
\underset{s \times 1}{d z}(t)=\underset{s \times 1}{\mu_{z}(t, z)} d t+\underset{s \times n}{(t, z)^{\prime}} \underset{n \times 1}{W}(t)
$$


where $z\left(t_{0}\right)$ is a deterministic vector that defines the initial state of the system, $W(t)$ is a vector of $n$ independent Wiener processes, ${ }^{1}$ and the prime denotes transposition. The usual properties for guaranteeing the existence of a strong solution to (1) are assumed to hold. The vector $z(t)$ can be divided into two components: the financial state variables $z_{f}(t)$ and the mortality intensity of a group of individuals, which, as customary in the actuarial literature, are assumed to be homogeneous by cohort:

$$
\underbrace{\left[\begin{array}{c}
d z_{f}(t) \\
(s-1) \times 1 \\
d \lambda(t)
\end{array}\right]}_{d z(t)}=\underbrace{\left[\begin{array}{c}
\mu_{z f}(t, z) \\
(s-1) \times 1 \\
\mu_{\lambda}(t, z)
\end{array}\right]}_{\mu_{z}(t, z)} d t+\underbrace{\left[\begin{array}{cc}
\Omega_{f}(t, z)^{\prime} & \mathbf{0} \\
(s-1) \times(n-1) & (s-1) \times 1 \\
\sigma_{f \lambda}(t, z)^{\prime} & \sigma_{\lambda}(t, z) \\
1 \times(n-1)
\end{array}\right]}_{\Omega(t, z)^{\prime}} \underbrace{\left[\begin{array}{c}
d W_{f}(t) \\
(n-1) \times 1 \\
d W_{\lambda}(t)
\end{array}\right]}_{d W(t)},
$$

where $\mathbf{0}$ is a vector of zeros. The diffusion vector $\sigma_{f \lambda}(t, z)$ captures the correlation between the financial state variables $z_{f}(t)$ and the mortality intensity $\lambda(t)$.

\subsection{Financial market}

On the financial market $n$ risky assets are traded. Their prices $S(t) \in \mathbb{R}_{+}^{n}$ solve the (matrix) stochastic differential equation

$$
\underset{n \times 1}{I_{S \times 1}^{-1}} \underset{n \times 1}{S} \underset{n \times 1}{\mu(t, z)} d t+\underset{n \times n}{(t, z)^{\prime}} \underset{n \times 1}{W} \underset{n \times 1}{(t)}
$$

where $I_{S}$ is a diagonal matrix containing the elements of vector $S(t)$. The initial asset prices $S\left(t_{0}\right)$ are deterministic. Finally, a risk-less asset exists, whose price $G(t) \in \mathbb{R}_{+}$

\footnotetext{
${ }^{1}$ The case with dependent Wiener processes can be easily obtained through the Cholesky's decomposition.
} 
solves the ordinary differential equation

$$
G(t)^{-1} d G(t)=r(t, z) d t
$$

where $r(t, z) \in \mathbb{R}_{+}$is the instantaneously risk-less interest rate. We assume $G\left(t_{0}\right)=1$, i.e. the risk-less asset is the numéraire of the economy. The financial market is assumed to be arbitrage free and complete. In other words, a unique vector of market prices of risk $\xi(t, z) \in \mathbb{R}^{n}$ exists, such that $\Sigma(t, z)^{\prime} \xi(t, z)=\mu(t, z)-r(t, z) \mathbf{1}$, where $\mathbf{1}$ is a vector of ones (i.e. $\left.\exists ! \Sigma(t, z)^{-1}\right)$.

Girsanov's theorem allows us to switch from the historical $(\mathbb{P})$ to the risk-neutral probability $\mathbb{Q}$ by using $d W^{\mathbb{Q}}(t)=\xi(t, z) d t+d W(t)$. The value in $t_{0}$ of any cash flow $\Xi(t)$ available at time $t$ can be written as

$$
\Xi\left(t_{0}\right)=\mathbb{E}_{t_{0}}^{\mathbb{Q}}\left[\Xi(t) \frac{G\left(t_{0}\right)}{G(t)}\right]=\mathbb{E}_{t_{0}}^{\mathbb{Q}}\left[\Xi(t) e^{-\int_{t_{0}}^{t} r(u, z) d u}\right]=\mathbb{E}_{t_{0}}\left[\Xi(t) m\left(t_{0}, t\right) e^{-\int_{t_{0}}^{t} r(u, z) d u}\right]
$$

where $\mathbb{E}_{t_{0}}[\bullet]$ and $\mathbb{E}_{t_{0}}^{\mathbb{Q}}[\bullet]$ are the expected value operators under the historical $(\mathbb{P})$ and the risk neutral $(\mathbb{Q})$ probabilities respectively, conditional on the information set at time $t_{0}$, and the martingale $m\left(t_{0}, t\right)$, such that $m\left(t_{0}, t_{0}\right)=1$, solves $m\left(t_{0}, t\right)^{-1} d m\left(t_{0}, t\right)=$ $-\xi(t, z) d W(t)$

\subsection{Longevity bonds market}

The mortality intensity (or force of mortality) $\lambda(t, z) \in \mathbb{R}_{+}$of a homogeneous group of individuals, which the investor belongs to, is an element of $z(t)$. Following the stochastic mortality approach initiated by Milevsky and Promislow (2001) and Dahl 
(2004), the death event is modelled as a Poisson process with stochastic intensity. The probability to be alive at time $t$, given that an agent is alive in $t_{0}$, is given by $\mathbb{E}_{t_{0}}^{\mathbb{P}}\left[e^{-\int_{t_{0}}^{t} \lambda(u, z) d u}\right]$.

The value in $t_{0}$ of a financial flow $\Xi(t)$ available in $t$ if an agent is still alive can be written as $\mathbb{E}_{t_{0}}^{\mathbb{Q}}\left[\Xi(t) e^{-\int_{t_{0}}^{t} r(u, z)+\lambda(u, z) d u}\right]$, while the value of the same cash flow available at the death time of an agent is given by $\mathbb{E}_{t_{0}}^{\mathbb{Q}}\left[\int_{t_{0}}^{\infty} \lambda(s) \Xi(s) e^{-\int_{t_{0}}^{s} r(u, z)+\lambda(u, z) d u} d s\right]$, where we have assumed that the death time is defined on the interval $\left[t_{0},+\infty[\right.$ (see Lando, 1998).

The financial market described in the previous section is assumed to be complete even with respect to the force of mortality. In other words, we assume that there exists a derivative on $\lambda(t)$, which we will refer to hereafter as the "longevity asset". If $\Lambda(t)$ is the price of such an asset, the dynamics of asset prices (3) can be rewritten disentangling the (independent) Wiener processes $W_{f}(t)$ and $W_{\lambda}(t)$ :

$$
\underbrace{\left[\begin{array}{cc}
I_{S_{f}}^{-1} & \mathbf{0} \\
(n-1) \times(n-1) & (n-1) \times 1 \\
\mathbf{0}_{1 \times(n-1)}^{\prime} & \Lambda^{-1}
\end{array}\right]}_{I_{S}^{-1}} \underbrace{\left[\begin{array}{c}
d S_{f}(t) \\
(n-1) \times 1 \\
d \Lambda(t)
\end{array}\right]}_{d S(t)}=\underbrace{\left[\begin{array}{c}
\mu_{f}(t, z) \\
(n-1) \times 1 \\
\mu_{\Lambda}(t, z)
\end{array}\right]}_{\mu(t, z)} d t+\underbrace{\left[\begin{array}{cc}
\Sigma_{f}(t, z)^{\prime} & \mathbf{0} \\
(n-1) \times(n-1) & (n-1) \times 1 \\
\sigma_{\Lambda f}(t, z)^{\prime} & \sigma_{\Lambda \lambda}(t, z) \\
1 \times(n-1)
\end{array}\right]}_{\Sigma(t, z)^{\prime}} \underbrace{\left[\begin{array}{c}
d W_{f}(t) \\
(n-1) \times 1 \\
d W_{\lambda}(t)
\end{array}\right]}_{d W(t)} .
$$

\section{Investor's maximisation problem}

\subsection{Investor's wealth, consumption and revenue}

The investor holds $\theta_{S}(t) \in \mathbb{R}^{n}$ units of the risky assets and $\theta_{G}(t) \in \mathbb{R}$ units of the risk-less asset. Thus, at any instant in time, the investor's wealth $R(t)$ is given by the 
static budget constraint

$$
R(t)=\theta_{S}(t)^{\prime} S(t)+\theta_{G}(t) G(t)
$$

whose differential is the dynamic budget constraint

$$
d R(t)=\theta_{S}(t)^{\prime} d S(t)+\theta_{G}(t) d G(t)+\underbrace{d \theta_{S}(t)^{\prime}(S(t)+d S(t))+d \theta_{G}(t) G(t)}_{d R_{a}(t)} .
$$

The first two components on the right hand side of (8) account for the changes in prices. The $d R_{a}(t)$ component, which accounts for the dynamic adjustment of the portfolio allocation: (i) finances the instantaneous consumption $c(t) d t$, (ii) discounts the probability of dying between $t$ and $t+d t$, which is measured by $\lambda(t, z) d t$, and (iii) is financed by the investor's labour income.

The accumulated labour income from $t_{0}$ up to time $t$ is $L(t)$ and solves

$$
d L(t)=w(t, z) d t+\underbrace{\left[\begin{array}{cc}
\sigma_{L f}(t, z)^{\prime} & \sigma_{L \lambda}(t, z)
\end{array}\right]}_{\sigma_{L}(t, z)^{\prime}} \underbrace{\left[\begin{array}{c}
d W_{f}(t) \\
(n-1) \times 1 \\
d W_{\lambda}(t)
\end{array}\right]}_{d W(t)},
$$

where $w(t, z)$ is the (instantaneous) labour income (or wage) of the agent. Thus, the investor's wealth dynamics is

$$
d R(t)=\theta_{S}(t)^{\prime} d S(t)+\theta_{G}(t) d G(t)-c(t) d t+d L(t)+\lambda(t, z) R(t) d t .
$$

Once the static budget constraint (7) and the asset differentials (3), (4) and (9) are 
suitably taken into account, $d R(t)$ becomes

$$
\begin{aligned}
d R(t)= & \left(R(t)(r(t, z)+\lambda(t, z))+\theta_{S}(t)^{\prime} I_{S}(\mu(t, z)-r(t, z) 1)+w(t, z)-c(t)\right) d t \\
& +\left(\theta_{S}(t)^{\prime} I_{S} \Sigma(t, z)^{\prime}+\sigma_{L}(t, z)^{\prime}\right) d W(t) .
\end{aligned}
$$

\subsection{Investor's preferences and objective}

The investor obtains utility from both the inter-temporal consumption $U_{c}(c(t))=$ $\left(c(t)-c_{m}\right)^{1-\delta} /(1-\delta)$ and the wealth at the end of the financial horizon $U_{R}(R(T))=$

$\left(R(T)-R_{m}\right)^{1-\delta} /(1-\delta)$, where $\delta>1$ and the constants $c_{m}$ and $R_{m}$ can be interpreted as the minimum subsistence value of consumption and final wealth, respectively. Both functions belong to the Hyperbolic Absolute Risk Aversion (HARA) family. In fact, the Arrow-Pratt absolute risk aversion indexes are $\delta /\left(c(t)-c_{m}\right)$ and $\delta /\left(R(T)-R_{m}\right)$, respectively. Accordingly, the higher $c_{m}$ (or $R_{m}$ ), the higher the risk aversion: an agent who has to guarantee a higher minimum level of consumption (or final wealth) will choose a safer investment. The case of CRRA preferences is obtained with $c_{m}=R_{m}=0$.

The investor chooses $\left(c(t), \theta_{S}(t)\right)$ which maximise the inter-temporal utility of his/her wealth and consumption up to time $T$ :

$\max _{\theta_{S}(t), c(t)} \mathbb{E}_{t_{0}}\left[\int_{t_{0}}^{T} \frac{\left(c(t)-c_{m}\right)^{1-\delta}}{1-\delta} e^{-\int_{t_{0}}^{s} \rho(u, z)+\lambda(u, z) d u} d t+\frac{\left(R(T)-R_{m}\right)^{1-\delta}}{1-\delta} e^{-\int_{t_{0}}^{T} \rho(u, z)+\lambda(u, z) d u}\right]$,

where $\rho(t, z)$ is a (possibly stochastic) subjective discount rate.

Notice that the budget constraint equalises the initial wealth increased by the expected value of all the future revenues to the sum between the final wealth and the 
whole consumption stream (recall $\left.\mathbb{E}_{t_{0}}^{\mathbb{Q}}[d L(t, z)]=\mathbb{E}_{t_{0}}^{\mathbb{Q}}\left[w(t, z)-\sigma_{L}(t, z)^{\prime} \xi(t, z)\right] d t\right)$ :

$R\left(t_{0}\right)=\mathbb{E}_{t_{0}}^{\mathbb{Q}}\left[\int_{t_{0}}^{T}\left(c(s)-w(s, z)+\sigma_{L}(s, z)^{\prime} \xi(s, z)\right) e^{-\int_{t_{0}}^{s} r(u, z)+\lambda(u, z) d u} d s+R(T) e^{-\int_{t_{0}}^{T} r(u, z)+\lambda(u, z) d u}\right]$,

i.e. the difference between the discounted value of final wealth at $T$ and the initial wealth of the investor must coincide with the expected value of the discounted flow of risk-adjusted consumption net of labour income.

\subsection{The optimal consumption and portfolio}

Problem (12) under the constraint (13) can be solved either through dynamic programming (via the so-called Hamilton-Jacobi-Bellman equation) or through the socalled martingale approach. This last method is viable in our framework because of market completeness.

Proposition 1. The optimal consumption and portfolio solving problem (12) are

$$
\begin{aligned}
c^{*}(t)= & c_{m}+\frac{R(t)-H(t, z)}{F(t, z)}, \\
I_{S} \theta_{S}^{*}(t)= & -\Sigma(t, z)^{-1} \sigma_{L}(t, z)+\frac{R(t)-H(t, z)}{\delta} \Sigma(t, z)^{-1} \xi(t, z) \\
& +\frac{R(t)-H(t, z)}{F(t, z)} \Sigma(t, z)^{-1} \Omega(t, z) \frac{\partial F(t, z)}{\partial z}+\Sigma(t, z)^{-1} \Omega(t, z) \frac{\partial H(t, z)}{\partial z},
\end{aligned}
$$

where

$H(t, z)=\mathbb{E}_{t}^{\mathbb{Q}}\left[\int_{t}^{T}\left(c_{m}-w(s, z)+\sigma_{L}(s, z)^{\prime} \xi(s, z)\right) e^{-\int_{t_{0}}^{s} r(u, z)+\lambda(u, z) d u} d s+R_{m} e^{-\int_{t_{0}}^{T} r(u, z)+\lambda(u, z) d u}\right]$, 


$$
\begin{gathered}
F(t, z)=\mathbb{E}_{t}^{\mathbb{Q}_{\delta}}\left[\begin{array}{c}
\int_{t}^{T} e^{-\int_{t}^{s}\left(\frac{\delta-1}{\delta} r(u, z)+\frac{1}{\delta} \rho(u, z)+\lambda(u, z)+\frac{1}{2} \frac{1}{\delta} \frac{\delta-1}{\delta} \xi(u, z)^{\prime} \xi(u, z)\right) d u} d s \\
+e^{-\int_{t}^{T}\left(\frac{\delta-1}{\delta} r(u, z)+\frac{1}{\delta} \rho(u, z)+\lambda(u, z)+\frac{1}{2} \frac{1}{\delta} \frac{\delta-1}{\delta} \xi(u, z)^{\prime} \xi(u, z)\right) d u}
\end{array}\right] \\
d W(t)^{\mathbb{Q}_{\delta}}=\frac{\delta-1}{\delta} \xi(t, z) d t+d W(t) .
\end{gathered}
$$

Proof. See A.

In the solution we used the new probability measure $\mathbb{Q}_{\delta}$ defined in (18). It has two relevant properties: (i) for a log-utility agent, i.e. $\delta=1$, the probability $\mathbb{Q}_{\delta}$ coincides with the historical probability; (ii) when the agent is infinitely risk averse, i.e. $\delta \rightarrow+\infty$, the probability $\mathbb{Q}_{\delta}$ coincides with $\mathbb{Q}$. In fact, we can think of the Wiener processes under $\mathbb{Q}_{\delta}$ as a weighted mean of the Wiener processes under the risk neutral and the historical probabilities:

$$
d W(t)^{\mathbb{Q}_{\delta}}=\left(1-\frac{1}{\delta}\right) d W(t)^{\mathbb{Q}}+\frac{1}{\delta} d W(t) .
$$

The function $H(t, z)$ is the expected value, under $\mathbb{Q}$, of the minimum final wealth $R_{m}$ and of the risk-adjusted minimum consumption level net of wage, appropriately discounted in both actuarial and financial terms. Thus, it represents the net expected balance after financing the minimum consumption and final wealth.

The function $F(t, z)$ is the expected value (under the preference-adjusted measure $\mathbb{Q}_{\delta}$ ) of the sum of all the discount factors for both the consumption stream (first term) and the final wealth (second term). We can interpret $F(t, z)$ as a "global" discount factor.

Optimal consumption is equal to the sum of the minimum consumption level $c_{m}$ and the difference between actual wealth $R(t)$ and the expected value of all the discounted 
subsistence levels of consumption and terminal wealth $H(t, z)$, divided by the "global" risk and preference-adjusted discount factor $F(t, z)$. We remark that the difference $R(t)-H(t, z)$ is also relevant for computing the optimal portfolio, which depends also on the sensitivities of $H(t, z)$ and $F(t, z)$ with respect to the state variables $z$.

The role of the longevity asset in (15) can be identified using the decomposition of matrices $\Omega$ and $\Sigma$ as shown in (2) and (6), respectively. The optimal investment in the longevity asset and in the financial assets is, respectively:

$$
\begin{gathered}
\Lambda \theta_{\Lambda}^{*}=-\frac{\sigma_{L \lambda}}{\sigma_{\Lambda \lambda}}+\frac{R-H}{\delta} \frac{1}{\sigma_{\Lambda \lambda}}\left(\xi_{\lambda}+\frac{\delta}{F} \sigma_{f \lambda}^{\prime} \frac{\partial F}{\partial z_{f}}+\sigma_{\lambda} \frac{\delta}{F} \frac{\partial F}{\partial \lambda}\right)-\frac{1}{\sigma_{\Lambda \lambda}}\left(\sigma_{f \lambda}^{\prime} \frac{\partial H}{\partial z_{f}}+\sigma_{\lambda} \frac{\partial H}{\partial \lambda}\right) \\
I_{S_{f}} \theta_{S_{f}}^{*}=-\Sigma_{f}^{-1} \sigma_{L f}+\frac{R-H}{\delta} \Sigma_{f}^{-1}\left(\xi_{f}+\frac{\delta}{F} \Omega_{f}^{\prime} \frac{\partial F}{\partial z_{f}}\right)-\Sigma_{f}^{-1} \Omega_{f}^{\prime} \frac{\partial H}{\partial z_{f}}-\Sigma_{f}^{-1} \sigma_{\Lambda f} \Lambda \theta_{\Lambda}^{*} .
\end{gathered}
$$

We identify four components in the demand for the longevity asset: (i) a speculative component, related to the risk premium $\xi_{\lambda}$, (ii) a hedging component against labour income fluctuations, (iii) a hedging component against the fluctuations of the global discount factor $F(t, z)$, and (iv) a hedging component against the fluctuations of the expected imbalance to finance minimum consumption and wealth $H(t, z)$.

The last two components depend on: (i) the risk aversion of the individual, (ii) the variance-covariance matrix of the state variables, and (iii) the sensitivities of $F(t, z)$ and $H(t, z)$ with respect to changes in the state variables.

The inclusion of the longevity asset in the investment set modifies the individual demand of both the risk-less and the other financial assets. We can interpret the amount of wealth invested in the longevity asset as taken partly from the wealth invested in the risk-less asset and partly from the wealth invested in the financial assets (as in 
Menoncin, 2008). The proportion taken from the financial assets is given by the ratio between the covariance of the longevity and the financial assets and the variance of the

financial assets (in fact, $\left.\left(\Sigma_{f}^{\prime} \Sigma_{f}\right)^{-1} \Sigma_{f}^{\prime} \sigma_{\Lambda f}=\Sigma_{f}^{-1} \sigma_{\Lambda f}\right)$. This means that the higher the correlation between a financial asset and the longevity asset, the higher the amount of wealth taken from the former to be invested in the latter. If the longevity asset is not correlated to financial assets (i.e. $\sigma_{\Lambda f}=0$ ), then the amount of money to be invested in it is fully taken from what is invested in the risk-less asset.

\section{A numerical application}

\subsection{The state variables}

In order to present a numerical application, a simplified market structure is taken into account. We consider two uncorrelated state variables: the instantaneously risk-less interest rate $r(t)$ and the force of mortality $\lambda(t)$. They solve the following differential equations

$$
\begin{gathered}
d r(t)=\alpha_{r}\left(\beta_{r}-r(t)\right) d t+\sigma_{r} \sqrt{r(t)} d W_{r}(t), \\
d \lambda(t)=\alpha_{\lambda}(\underbrace{\frac{1}{\alpha_{\lambda}} \frac{\partial \gamma(t)}{\partial t}+\gamma(t)}_{\beta_{\lambda}(t)}-\lambda(t)) d t+\sigma_{\lambda} \sqrt{\lambda(t)} d W_{\lambda}(t),
\end{gathered}
$$

where $\alpha_{r}, \alpha_{\lambda}>0$ are the strength of the mean reversion effect and $\beta_{r}, \gamma(t)>0$ are the long-term means. $\gamma(t)$ is set to be equal to the Gompertz law (with $\lambda\left(t_{0}\right)=\gamma\left(t_{0}\right)$, see Menoncin, 2009). If the Feller conditions $2 a_{r} \beta_{r}>\sigma_{r}^{2}$ and $2 \alpha_{\lambda} \beta_{\lambda}(t)>\sigma_{\lambda}^{2}$ are satisfied for any $t$, the two processes are always positive.

In order to preserve the statistical properties of $r(t)$ and $\lambda(t)$ after switching 
between probabilities, we assume that both the market prices of interest rate risk and mortality risk are proportional to the square root of the respective variable $\left(\phi_{r}\right.$ and $\phi_{\lambda}$ are constant):

$$
\xi_{r}=\phi_{r} \sqrt{r(t)}, \quad \xi_{\lambda}=\phi_{\lambda} \sqrt{\lambda(t)}
$$

The results we are about to present rely on the following proposition.

Proposition 2. If the variable $y(t)$ solves the stochastic differential equation

$$
d y(t)=\alpha(\beta(t)-y(t)) d t+\sigma \sqrt{y(t)} d W(t)
$$

then, for any constant $\chi$,

$$
\begin{aligned}
\mathbb{E}_{t}\left[e^{-\chi \int_{t}^{T} y(u) d u}\right] & =e^{-\alpha \int_{t}^{T} \beta(s) C(s ; \chi, \alpha, \sigma, T) d s-C(t ; \chi, \alpha, \sigma, T) y(t)}, \\
C(t ; \chi, \alpha, \sigma, T) & =2 \chi \frac{1-e^{-\Delta(T-t)}}{\Delta+\alpha+(\Delta-\alpha) e^{-\Delta(T-t)}} \\
\Delta & \equiv \sqrt{\alpha^{2}+2 \sigma^{2} \chi}
\end{aligned}
$$

Proof. See Appendix B.

In order to simplify the computations, we assume that the labour income is deterministic (i.e. $\sigma_{L}=0$ ) and the wage $w$ is constant. Thus, the function $H(t, z)$ can be written as

$H(t, z)=\left(c_{m}-w\right) \int_{t}^{T} \mathbb{E}_{t}^{\mathbb{Q}}\left[e^{-\int_{t}^{s} r(u, z) d u}\right] \mathbb{E}_{t}^{\mathbb{Q}}\left[e^{-\int_{t}^{s} \lambda(u, z) d u}\right] d s+R_{m} \mathbb{E}_{t}^{\mathbb{Q}}\left[e^{-\int_{t}^{T} r(u, z) d u}\right] \mathbb{E}_{t}^{\mathbb{Q}}\left[e^{-\int_{t}^{T} \lambda(u, z) d u}\right]$ 
where

$$
\begin{gathered}
\mathbb{E}_{t}^{\mathbb{Q}}\left[e^{-\int_{t}^{T} r(u) d u}\right]=e^{-\alpha_{r}^{\mathbb{Q}} \beta_{r}^{\mathbb{Q}} \int_{t}^{T} C\left(s ; 1, \alpha_{r}^{\mathbb{Q}}, \sigma_{r}, T\right) d s-C\left(t ; 1, \alpha_{r}^{\mathbb{Q}}, \sigma_{r}, T\right) r(t)}, \\
\mathbb{E}_{t}^{\mathbb{Q}}\left[e^{-\int_{t}^{T} \lambda(u) d u}\right]=e^{-\alpha_{\lambda}^{\mathbb{Q}} \int_{t}^{T} \beta_{\lambda}^{\mathbb{Q}}(s) C\left(s ; 1, \alpha_{\lambda}^{\mathbb{Q}}, \sigma_{\lambda}, T\right) d s-C\left(t ; 1, \alpha_{\lambda}^{\mathbb{Q}}, \sigma_{\lambda}, T\right) \lambda(t)} .
\end{gathered}
$$

\subsection{The traded assets}

Four assets are traded on the financial market:

- the risk-less asset, whose price $G(t)$ evolves as in (4);

- a risky asset (like a stock index) whose price $A(t)$ follows a GBM:

$$
A(t)^{-1} d A(t)=\mu d t+\sigma_{A} d W_{A}(t)+\sigma_{A r} d W_{r}(t)
$$

where $\sigma_{A r}$ measures the instantaneous covariance between the stock and the riskless interest rate. We assume $\xi_{A}$ is constant and thus

$$
\begin{aligned}
A(t)^{-1} d A(t) & =r(t) d t+\sigma_{A} d W_{A}^{\mathbb{Q}}(t)+\sigma_{A r} d W_{r}^{\mathbb{Q}}(t) \\
& =\left(r(t)+\sigma_{A} \xi_{A}+\sigma_{A r} \phi_{r} \sqrt{r(t)}\right) d t+\sigma_{A} d W_{A}(t)+\sigma_{A r} d W_{r}(t)
\end{aligned}
$$

- a constant time to maturity $\left(T_{B}\right)$ zero-coupon bond whose price is

$$
B(t)=\mathbb{E}_{t}^{\mathbb{Q}}\left[e^{-\int_{t}^{t+T_{B}} r(u) d u}\right]
$$


and whose differential is

$$
\begin{aligned}
B(t)^{-1} d B(t)= & r(t) d t-C\left(0 ; 1, \alpha_{r}^{\mathbb{Q}}, \sigma_{r}, T_{B}\right) \sigma_{r} \sqrt{r(t)} d W_{r}^{\mathbb{Q}}(t) \\
= & r(t)\left(1-C\left(0 ; 1, \alpha_{r}^{\mathbb{Q}}, \sigma_{r}, T_{B}\right) \sigma_{r} \phi_{r}\right) d t \\
& -C\left(0 ; 1, \alpha_{r}^{\mathbb{Q}}, \sigma_{r}, T_{B}\right) \sigma_{r} \sqrt{r(t)} d W_{r}(t) ;
\end{aligned}
$$

- a constant time to maturity $\left(T_{\Lambda}\right)$ zero-coupon longevity bond whose price is

$$
\Lambda(t)=\mathbb{E}_{t}^{\mathbb{Q}}\left[e^{-\int_{t}^{t+T_{\Lambda}} r(u)+\lambda(u) d u}\right]
$$

and whose differential is

$$
\begin{aligned}
\Lambda(t)^{-1} d \Lambda(t)= & (r(t)+\lambda(t)) d t-C\left(0 ; 1, \alpha_{r}^{\mathbb{Q}}, \sigma_{r}, T_{\Lambda}\right) \sigma_{r} \sqrt{r(t)} d W_{r}^{\mathbb{Q}}(t) \\
& -C\left(0 ; 1, \alpha_{\lambda}^{\mathbb{Q}}, \sigma_{\lambda}, T_{\Lambda}\right) \sigma_{\lambda} \sqrt{\lambda(t)} d W_{\lambda}^{\mathbb{Q}}(t) \\
= & \left(\left(1-C\left(0 ; 1, \alpha_{r}^{\mathbb{Q}}, \sigma_{r}, T_{\Lambda}\right) \sigma_{r} \phi_{r}\right) r(t)+\left(1-C\left(0 ; 1, \alpha_{\lambda}^{\mathbb{Q}}, \sigma_{\lambda}, T_{\Lambda}\right) \sigma_{\lambda} \phi_{\lambda}\right) \lambda(t)\right) d t \\
& -C\left(0 ; 1, \alpha_{r}^{\mathbb{Q}}, \sigma_{r}, T_{\Lambda}\right) \sigma_{r} \sqrt{r(t)} d W_{r}(t)-C\left(0 ; 1, \alpha_{\lambda}^{\mathbb{Q}}, \sigma_{\lambda}, T_{\Lambda}\right) \sigma_{\lambda} \sqrt{\lambda(t)} d W_{\lambda}(t) .
\end{aligned}
$$

Since the market price of the stock $\left(\xi_{A}\right)$ is constant then the function $F(t)$ can be written as

$$
\begin{aligned}
F(t)= & \int_{t}^{T} e^{-\left(\frac{1}{\delta} \rho+\frac{1}{2} \frac{1}{\delta} \frac{\delta-1}{\delta} \xi_{A}^{2}\right)(s-t)} \mathbb{E}_{t}^{\mathbb{Q}_{\delta}}\left[e^{-\frac{\delta-1}{\delta}\left(1+\frac{1}{2} \frac{1}{\delta} \phi_{r}^{2}\right) \int_{t}^{s} r(u) d u}\right] \mathbb{E}_{t}^{\mathbb{Q}_{\delta}}\left[e^{-\left(1+\frac{1}{2} \frac{1}{\delta} \frac{\delta-1}{\delta} \phi_{\lambda}^{2}\right) \int_{t}^{s} \lambda(u) d u}\right] d s \\
& +e^{-\left(\frac{1}{\delta} \rho+\frac{1}{2} \frac{1}{\delta} \frac{\delta-1}{\delta} \xi_{A}^{2}\right)(T-t)} \mathbb{E}_{t}^{\mathbb{Q}_{\delta}}\left[e^{-\frac{\delta-1}{\delta}\left(1+\frac{1}{2} \frac{1}{\delta} \phi_{r}^{2}\right) \int_{t}^{T} r(u) d u}\right] \mathbb{E}_{t}^{\mathbb{Q}_{\delta}}\left[e^{-\left(1+\frac{1}{2} \frac{1}{\delta} \frac{\delta-1}{\delta} \phi_{\lambda}^{2}\right) \int_{t}^{T} \lambda(u) d u}\right](38)
\end{aligned}
$$


where

$$
\begin{aligned}
& \mathbb{E}_{t}^{\mathbb{Q}_{\delta}}\left[e^{-\frac{\delta-1}{\delta}\left(1+\frac{1}{2} \frac{1}{\delta} \phi_{r}^{2}\right) \int_{t}^{s} r(u) d u}\right]=e^{-\alpha_{r}^{\mathbb{Q}_{\delta} \delta} \beta_{r}^{\mathbb{Q}_{\delta}} \int_{t}^{s} C\left(u ; \frac{\delta-1}{\delta}\left(1+\frac{1}{2} \frac{1}{\delta} \phi_{r}^{2}\right), \alpha_{r}^{\mathbb{Q}_{\delta}}, \sigma_{r}, s\right) d u-C\left(t ; \frac{\delta-1}{\delta}\left(1+\frac{1}{2} \frac{1}{\delta} \phi_{r}^{2}\right), \alpha_{r}^{\mathbb{Q}_{\delta}}, \sigma_{r}, s\right) r(t)}, \\
& \mathbb{E}_{t}^{\mathbb{Q}_{\delta}}\left[e^{-\left(1+\frac{1}{2} \frac{1}{\delta} \frac{\delta-1}{\delta} \phi_{\lambda}^{2}\right) \int_{t}^{s} \lambda(u) d u}\right]=e^{-\alpha_{\lambda}^{\mathbb{Q}_{\delta}} \int_{t}^{s} \beta_{\lambda}^{\mathbb{Q}_{\delta}}(s) C\left(u ;\left(1+\frac{1}{2} \frac{1}{\delta} \frac{\delta-1}{\delta} \phi_{\lambda}^{2}\right), \alpha_{\lambda}^{\mathbb{Q}_{\delta}}, \sigma_{\lambda}, s\right) d u-C\left(t ;\left(1+\frac{1}{2} \frac{1}{\delta} \frac{\delta-1}{\delta} \phi_{\lambda}^{2}\right), \alpha_{\lambda}^{\mathbb{Q}_{\delta}}, \sigma_{\lambda}, s\right) \lambda(t)} .
\end{aligned}
$$

\subsection{The optimal portfolio}

In order to explicitly compute the optimal portfolio from (15), we define the following matrices: $\theta_{S}(t)=\left[\begin{array}{lll}\theta_{A}(t) & \theta_{B}(t) & \theta_{\Lambda}(t)\end{array}\right]^{\prime}, d W(t)=\left[\begin{array}{lll}d W_{A}(t) & d W_{r}(t) & d W_{\lambda}(t)\end{array}\right]^{\prime}$, $z(t)=\left[\begin{array}{ll}r(t) & \lambda(t)\end{array}\right]^{\prime}, \xi=\left[\begin{array}{lll}\xi_{A} & \phi_{r} \sqrt{r(t)} & \phi_{\lambda} \sqrt{\lambda(t)}\end{array}\right]^{\prime}$,

$$
\begin{aligned}
& I_{S}=\left[\begin{array}{ccc}
A(t) & 0 & 0 \\
0 & B(t) & 0 \\
0 & 0 & \Lambda(t)
\end{array}\right], \Omega^{\prime}=\left[\begin{array}{ccc}
0 & \sigma_{r} \sqrt{r(t)} & 0 \\
0 & 0 & \sigma_{\lambda} \sqrt{\lambda(t)}
\end{array}\right]
\end{aligned}
$$

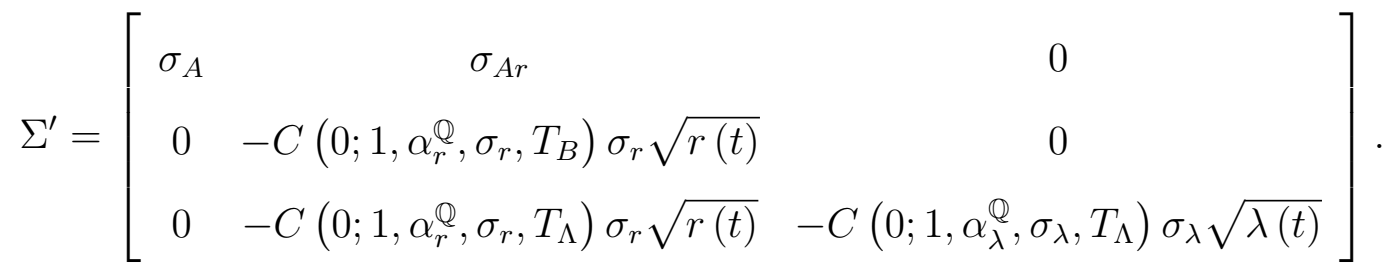


The optimal portfolio is

$$
\begin{aligned}
& {\left[\begin{array}{c}
A(t) \theta_{A}(t) \\
B(t) \theta_{B}(t) \\
\Lambda(t) \theta_{\Lambda}(t)
\end{array}\right]=\frac{R(t)-H(t)}{\delta}\left[\begin{array}{c}
\frac{\xi_{A}}{\sigma_{A}} \\
\frac{1}{C\left(0 ; 1, \alpha_{r}^{\Phi}, \sigma_{r}, T_{B}\right)}\left(\frac{\sigma_{A r} \xi_{A}}{\sigma_{A} \sigma_{r} \sqrt{r(t)}}-\frac{\phi_{r}}{\sigma_{r}}+\frac{\phi_{\lambda} C\left(0 ; 1, \alpha_{r}^{\otimes}, \sigma_{r}, T_{\Lambda}\right)}{\sigma_{\lambda} C\left(0 ; 1, \alpha_{\lambda}^{\Phi}, \sigma_{\lambda}, T_{\Lambda}\right)}\right) \\
-\frac{\phi_{\lambda}}{\sigma_{\lambda} C\left(0 ; 1, \alpha_{\lambda}, \sigma_{\lambda}, T_{\Lambda}\right)}
\end{array}\right]} \\
& +\frac{R(t)-H(t)}{F(t)}\left[\begin{array}{c}
0 \\
\frac{1}{C\left(0 ; 1, \alpha_{r}^{Q}, \sigma_{r}, T_{B}\right)}\left(-\frac{\partial F(t)}{\partial r(t)}+\frac{C\left(0 ; 1, \alpha_{r}^{\varrho}, \sigma_{r}, T_{\Lambda}\right)}{C\left(0 ; 1, \alpha_{\lambda}^{\varrho}, \sigma_{\lambda}, T_{\Lambda}\right)} \frac{\partial F(t)}{\partial \lambda(t)}\right) \\
-\frac{1}{C\left(0 ; 1, \alpha_{\lambda}^{\varrho}, \sigma_{\lambda}, T_{\Lambda}\right)} \frac{\partial F(t)}{\partial \lambda(t)}
\end{array}\right] \\
& +\left[\begin{array}{c}
0 \\
\frac{1}{C\left(0 ; 1, \alpha_{r}^{\varrho}, \sigma_{r}, T_{B}\right)}\left(-\frac{\partial H(t)}{\partial r(t)}+\frac{C\left(0 ; 1, \alpha_{r}^{Q}, \sigma_{r}, T_{\Lambda}\right)}{C\left(0 ; 1, \alpha_{\lambda}^{\varrho}, \sigma_{\lambda}, T_{\Lambda}\right)} \frac{\partial H(t)}{\partial \lambda(t)}\right) \\
-\frac{1}{C\left(0 ; 1, \alpha_{\lambda}^{\varrho}, \sigma_{\lambda}, T_{\Lambda}\right)} \frac{\partial H(t)}{\partial \lambda(t)}
\end{array}\right] .
\end{aligned}
$$

The optimal investment in the risk-less asset is obtained as the difference between the total wealth $R(t)$ and the amount invested in the other assets.

\subsection{Calibration}

All the financial market parameters are estimated from three time series (from January 1st, 1962 to January 1st, 2007, thus taking out the turbulence following the sub-prime crisis): (i) the 3-month US Treasury Bill interest rate (on secondary market) for calibrating $r(t)$, (ii) the 10-year US Bond interest rate (on secondary market) for calibrating $B(t)$ (with $T_{B}=10$ ), and (iii) S\&P 500 for calibrating $A(t)$.

The parameters of the risk-free interest rate $\alpha_{r}, \beta_{r}$ and $\sigma_{r}$ are gathered in Table 1 (they are calibrated by applying the homoscedastic transformation $2 \sqrt{r}(t)$ to $(22)$ and then using ordinary least squares). $r\left(t_{0}\right)$ is set to its long term equilibrium value (i.e. $\left.r_{0}=\beta_{r}\right)$. 
The average return on 10 -year bonds is about $7.1 \%$, thus

$$
\begin{gathered}
\mathbb{E}_{t}[d \ln B(t)]=0.071 d t \\
\left(1-C\left(0 ; 1, \alpha_{r}^{\mathbb{Q}}, \sigma_{r}, T_{B}\right) \sigma_{r} \phi_{r}-\frac{1}{2} C\left(0 ; 1, \alpha_{r}^{\mathbb{Q}}, \sigma_{r}, T_{B}\right)^{2} \sigma_{r}^{2}\right) r(t)=0.071
\end{gathered}
$$

which is solved for $\phi_{r}=-0.5590635$ (recall that $\alpha_{r}^{\mathbb{Q}}$ is a function of $\phi_{r}$ ) where instead of $r(t)$ we use the long term equilibrium value $\beta_{r}$ (Table 1 ).

The variance and the mean of the log-return of S\&P 500 are

$$
\begin{gathered}
\mathbb{V}[d \ln A(t)]=\left(\sigma_{A r}^{2}+\sigma_{A}^{2}\right) d t=0.0223 d t \\
\mathbb{E}[d \ln A(t)]=\left(r(t)+\sigma_{A} \xi_{A}+\sigma_{A r} \phi_{r} \sqrt{r(t)}-\frac{1}{2}\left(\sigma_{A}^{2}+\sigma_{A r}^{2}\right)\right) d t=0.06688 d t
\end{gathered}
$$

where $r(t)$ will be substituted with the long term equilibrium level $\beta_{r}$.

The covariance between the S\&P 500 log-return and the return on the 10-year bonds is

$$
\mathbb{C}[d \ln A(t), d \ln B(t)]=-C\left(0 ; 1, \alpha_{r}^{\mathbb{Q}}, \sigma_{r}, T_{B}\right) \sigma_{A r} \sigma_{r} \sqrt{r(t)} d t=-0.0004552 d t,
$$

where the interest rate will be again substituted with its long term mean $\beta_{r}$. Thus, we 
have to solve the following system

$$
\left\{\begin{array}{l}
\sigma_{A r}^{2}+\sigma_{A}^{2}=0.0223 \\
\beta_{r}+\sigma_{A} \xi_{A}+\sigma_{A r} \phi_{r} \sqrt{\beta_{r}}-\frac{1}{2}\left(\sigma_{A}^{2}+\sigma_{A r}^{2}\right)=0.06688 \\
-C\left(0 ; 1, \alpha_{r}^{\mathbb{Q}}, \sigma_{r}, T_{B}\right) \sigma_{A r} \sigma_{r} \sqrt{\beta_{r}}=-0.0004552,
\end{array}\right.
$$

which has a positive and a negative solution for $\sigma_{A}$; we take the positive one as shown in Table 1.

Mortality is estimated by fitting the observed survival probabilities for US males born in 1950, who were aged $60\left(t_{0}\right)$ at January 1st, 2010. We fix the observation point to January 1st, 1990 and we obtain the observed survival curve from cohort tables available at the Human Mortality Database using 20 data points. For $t \in\{1,2, \ldots, 19\}$ we get the survival probability $p(0, t)$. We calibrate the intensity process by minimising the mean squared error between fitted and observed values of the survival probability, imposing at the same time the Feller condition. The initial value of the mortality intensity is

$$
\lambda\left(t_{0}\right)=\phi_{0}+\frac{1}{b}\left(1+\frac{1}{\alpha_{\lambda}}\right) e^{\frac{t_{0}-m}{b}} .
$$

The survival probability between $t_{0}$ and $T$ is available in semi-closed form:

$$
\hat{p}\left(t_{0}, T\right)=\mathbb{E}_{t_{0}}^{\mathbb{P}}\left[e^{-\int_{t_{0}}^{T} \lambda(u) d u}\right]=e^{-\alpha_{\lambda} \int_{t_{0}}^{T} \beta_{\lambda}(s) C\left(s ; 1, \alpha_{\lambda}, \sigma_{\lambda}, T\right) d s-C\left(0 ; 1, \alpha_{\lambda}, \sigma_{\lambda}, T\right) \lambda\left(t_{0}\right)} .
$$

We thus determine the parameters of the intensity process (gathered in Table 1), 
Table 1: Parameters for the base scenario, calibrated on the S\&P 500, 3-month Treasury Bills, and 10-year Bonds time series (between January 1st 1962 and January 1st 2007).

\begin{tabular}{cccc}
\hline Interest rate/Bond & Stock & Wealth/Preferences & Mortality/Longevity \\
\hline$\alpha_{r}=0.0904668$ & $\sigma_{A}=0.14926$ & $R_{0}=100$ & $\alpha_{\lambda}=0.561$ \\
$\beta_{r}=0.0621328=r_{0}$ & $\sigma_{A r}=0.0046306$ & $w=10$ & $\sigma_{\lambda}=0.0352$ \\
$\sigma_{r}=0.0543625$ & $\xi_{A}=0.1108301$ & $T=65$ & $\phi_{0}=0.0009944$ \\
$\phi_{r}=-0.5590635$ & & $\rho=0.01$ & $b=12.9374$ \\
$T_{B}=10$ & $R_{m}=100$ & $m=86.4515$ \\
& $c_{m}=0$ & $t_{0}=60$ \\
& $\delta=2.5$ & $T_{L}=10$ \\
\hline
\end{tabular}

$\alpha_{\lambda}, \sigma_{\lambda}, \phi_{0}, b$ and $m$, by minimising the cost function

$$
\frac{1}{n} \sqrt{\sum_{t=1}^{n}(\hat{p}(0, t)-p(0, t))^{2}} .
$$

\subsection{Base scenario}

We assume the existence of a continuously-rolled over longevity bond with maturity $T_{L}=10$ years whose underlying is the mortality intensity of the cohort of US males born in 1950. In our base scenario, we set the risk premium for mortality $\phi_{\lambda}=0$. We consider an individual who, at the time horizon $T$, does not want to deplete all his initial wealth $R_{0}$. Instead, we assume he wants to reach a minimum amount of wealth $\left(R_{m}\right)$ at $T$, which will be annuitised to finance post-retirement consumption. Hence, we set $R_{m}=R_{0}=100$. We set the annual salary to one tenth of the initial wealth, i.e $w=10$. We fix retirement age at $T=65$ years.

A sample consumption/investment profile is presented in Figure 1, where the optimal consumption/investment is represented at daily intervals. The individual's wealth volatility is decreasing over time. At the beginning of the horizon, he invests around 
$22 \%$ in the stock, goes short $9 \%$ in the risk-free asset and $1.5 \%$ in the bond, and invests $88 \%$ in the longevity asset. While he approaches retirement, he progressively reduces his investment in equity, in order to reach his final wealth target with a higher degree of certainty, and increases the share invested in bonds and in the risk-free asset, which reaches almost 100\% approaching retirement age. For the same reason, he reduces consumption, which drops from around $15 \%$ at $t_{0}$ to around $7.5 \%$ at $T$. This drop is consistent across simulations, though its magnitude varies. Investment in the longevity asset is decreasing over time as well, mainly because over time the fluctuations of the discount factors due to changes in mortality likelihood affect less and less the valuation of wealth of the individual. Indeed, since $\phi_{\lambda}=0$, the demand for the longevity asset is entirely due to the hedging component against the fluctuations of the interest rate and of the functions $F$ and $H$.

Figure 2, which collects 100 paths with optimal strategies computed at monthly intervals, allows to appreciate the variability in sample paths. Investment profiles are very much stable across different paths, while the consumption profile is more volatile, as hinted above. This result is consistent with the findings of some works that indicate how consumption tends to absorb most of the uncertainty in models (Bernasconi et al., 2015, for instance, demonstrate that even uncertainty on fiscal parameters affects consumption but does not alter the decision to evade). Thus, after a financial shock, an agent prefers to vary his consumption rather than his portfolio allocation.

\subsection{Sensitivity analysis: longevity risk premium}

Given the absence of a liquid market for longevity securities at present, estimating a reasonable value for the risk premium is very difficult. The risk premium should be 
negative, as longevity bonds negatively react to changes in $\lambda(t)$, for which investors would require a compensation (a lower price for the zero-coupon bond). Here, we take a kind of worst-case scenario with $\phi_{\lambda}=0.1$ to provide a lower bound to the demand for longevity bonds in optimal individual portfolios. Figure 3 reports the optimal consumption and investment profiles in this case. While investment in the stock and the risk-less asset remain unchanged w.r.t. the base scenario, there is a strong re-allocation between longevity asset and bond (even if their behaviour over

time is very similar). The bond has a positive share, which is mainly taken from the wealth previously invested in the longevity asset, which now has a humped-shaped profile, increasing up to almost $50 \%$ from the initial $40.5 \%$ before decreasing to reach even negative values $(-6.6 \%)$ at retirement.

Obviously, any negative value for the risk premium increases the investment in the longevity asset. We performed the analysis by fixing $\phi_{\lambda}=-0.25$, a value which was suggested as reasonable by Loeys et al. (2007) and that lies in the range of estimates obtained by Bauer et al. (2010). The simulations show a pattern which is similar to the base scenario, but the investment choices are tilted towards the longevity asset, whose "speculative" attractiveness adds to the hedging motive. Initial investment reaches more than 200\%, and the additional allocation in the longevity asset is financed by short selling the risk-less asset.

\subsection{Sensitivity analysis: individual characteristics}

We present here an analysis of the changes in the consumption/portfolio choices due to: (i) the sex of the consumer/investor, (ii) the time horizon $(T)$, (iii) the risk aversion $(\delta)$, (iv) the subsistence final wealth $\left(R_{m}\right)$, and $(\mathrm{v})$ the subsistence consumption $\left(c_{m}\right)$. 
Table 2: Calibrated parameters of the mortality processes of individuals outside the base case scenario.

\begin{tabular}{ccc}
\hline Parameter & 60 -yr old females & 55 -year old males \\
\hline$\alpha_{\lambda}$ & 5.6863 & 0.5659 \\
$\sigma_{\lambda}$ & 0.0277 & 0.0243 \\
$\phi_{0}$ & $6.12 \cdot 10^{-17}$ & 0.0002 \\
$b$ & 13.0579 & 17.0836 \\
$m$ & 91.9838 & 90.9065 \\
\hline
\end{tabular}

Sex

We calibrate the stochastic mortality model to 60-year old US females, using the same procedure we described in Section 4.4, and we consider the longevity asset to be written on this mortality intensity. Parameters are reported in Table 2. Women should invest a higher fraction of their wealth in the longevity asset (due to their longer lifetime expectancy). The optimal share invested in this asset lies between $99 \%$ and $102 \%$ for the first 4 years of the horizon and drops only at the very end to reach $37 \%$ in the last month prior to retirement. This is due to the higher predictability of mortality rates, due to the females' higher level of mean reversion to the Gompertz law, which is due to the lower variability of mortality rates displayed by females in the calibrated part of the curve (survival rates from age 40 to 60 ).

\section{Time horizon}

Analysis of the consumption/investment profile of a younger individual, who is 55 at time $t_{0}$ (parameters are reported in Table 2), with same fixed retirement age at 65, reveals that demand of the longevity asset is above around $90 \%$ and slightly increasing, from age 55 to 60 . Consumption/investment decisions in the second part of the time period (when the agent is aged 60 to 65 ) are in line with the base scenario. In the 
first 3-year period, instead, consumption stays on average almost constant, and starts decreasing markedly around age 58. Investment in equity decreases in time, while the share of wealth in the risk-free asset increases, from $-55 \%$ to almost $100 \%$. Investment in the bond market is positive in the first 5 years, but drops from the initial $34.5 \%$ down to around $-30 \%$ before increasing in the last two years of the horizon.

\section{Risk aversion}

A smaller risk aversion $(\delta=1.5)$ obviously increases investment in the stock, up to an initial 37\%. Also, it slightly increases investment in the longevity asset over the whole horizon, with initial share at $88.6 \%$ (w.r.t. $88.1 \%$ in the base scenario). On the contrary, a higher risk aversion $(\delta=5)$ decreases investment in the stock (down to $11 \%$ at time $\left.t_{0}\right)$ and in the longevity asset (87.7\%). Apart from absolute values, investment and consumption profiles approaching retirement are similar to the base scenario.

\section{Subsistence final wealth}

When investor has no interest in setting a minimum level of final wealth (i.e. $R_{m}=$ 0) Figure 4 shows that consumption progressively increases over time and reaches $100 \%$ of wealth at retirement. Also, the investment profile is riskier, as the individual invests initially $42 \%$ in the stock and around $30 \%$ in the bond. Investment in the longevity asset is slightly reduced with respect to the base scenario, accounting initially for $71 \%$ of wealth. The agent finances his investments by short selling the risk-less asset.

\section{Subsistence consumption}

When the agent wants to set a minimum consumption level $\left(c_{m}=8\right.$, i.e. $8 \%$ of

his initial wealth, $2 \%$ less than his deterministic labour income flow) he assumes a 
more conservative behaviour. The initial investment in stock $(12 \%)$ and bond $(-16 \%)$ are both lowered, while the share in the risk-less asset $(18 \%)$ is increased w.r.t. the base scenario. Investment in the longevity asset is almost insensitive, slightly lowered, but still accounts for $86.5 \%$ of the initial wealth. Over time, the optimal portfolio qualitatively behaves as in the base scenario.

\section{Concluding comments}

This paper derives the optimal consumption and investment profiles of an individual prior to a fixed retirement age in the presence of longevity risk. The market is complete and a derivative on the mortality intensity of the individual is listed. Investment in such an asset is driven by three motivations: (i) speculation, if the risk premium is attractive, (ii) diversification, if the asset is negatively correlated with other financial assets, and (iii) hedging, as changes in mortality affect the discount factors of the individual. When focusing on this latter factor, we calibrate our model to US real data, and find that the demand for longevity asset might be relevant. The result is robust across different individual types. We explore sensitivity with respect to mortality risk premium, finding that the demand for the longevity asset is positive even when the risk premium is comparable to the one required for stocks. Our results seem to suggest that, alongside reinsurance of longevity risk, which is currently growing in volume and number of transactions, there is potential room for involving individual investors in the longevity market.

Here, we have numerically computed the relevance of a zero-coupon longevity bond, but the optimal design of longevity securities needs to be investigated more deeply (as 
suggested by Cocco and Gomes (2012)). Further research is needed as well in order to explore the impact of basis risk, i.e. the possibility that the longevity asset is imperfectly correlated with the mortality intensity of the individual. 
Figure 1: Sample path of optimal portfolio with the values gathered in Table 1.
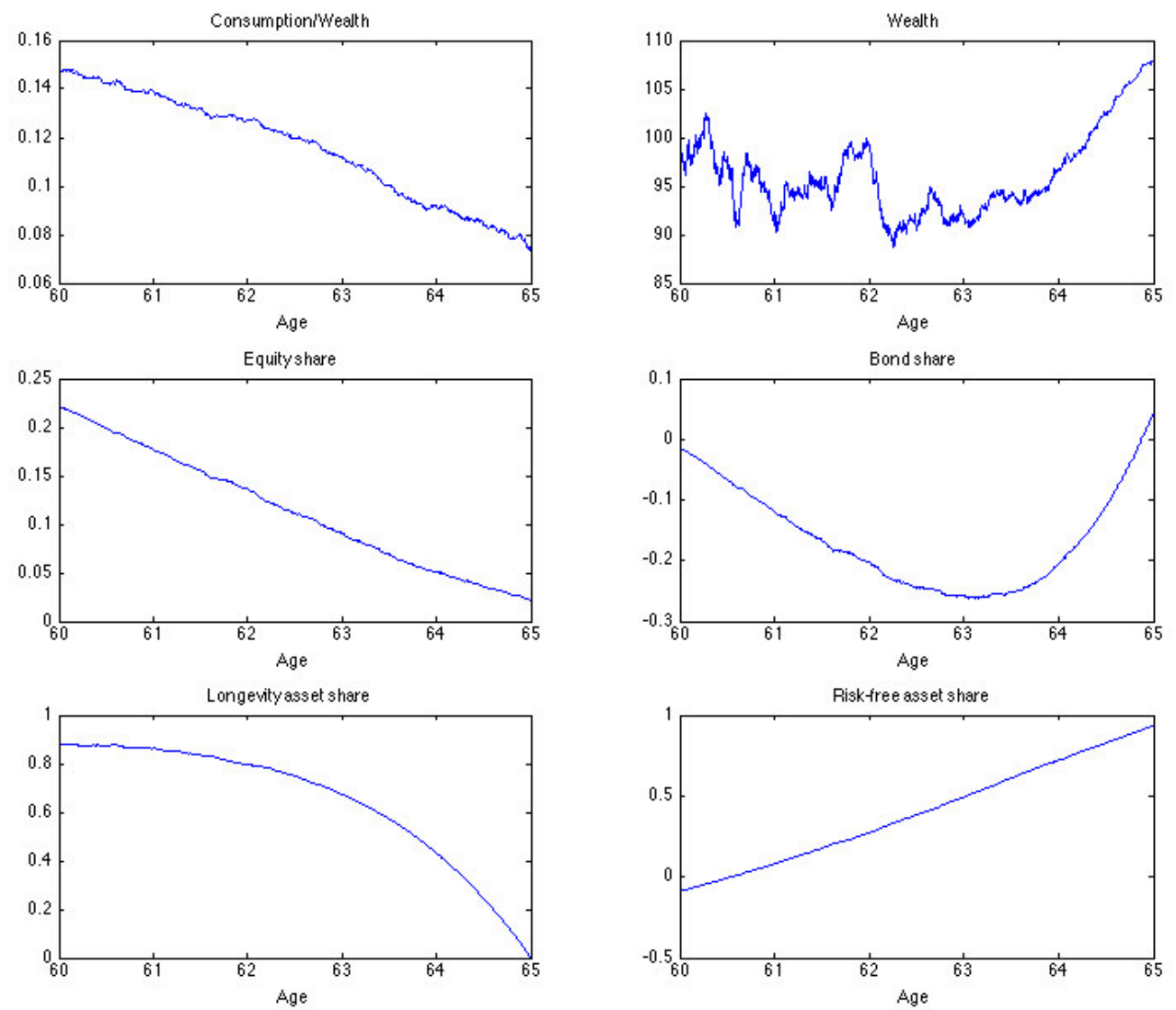
Figure 2: Simulation of optimal portfolio with the values gathered in Table 1, 100 paths.
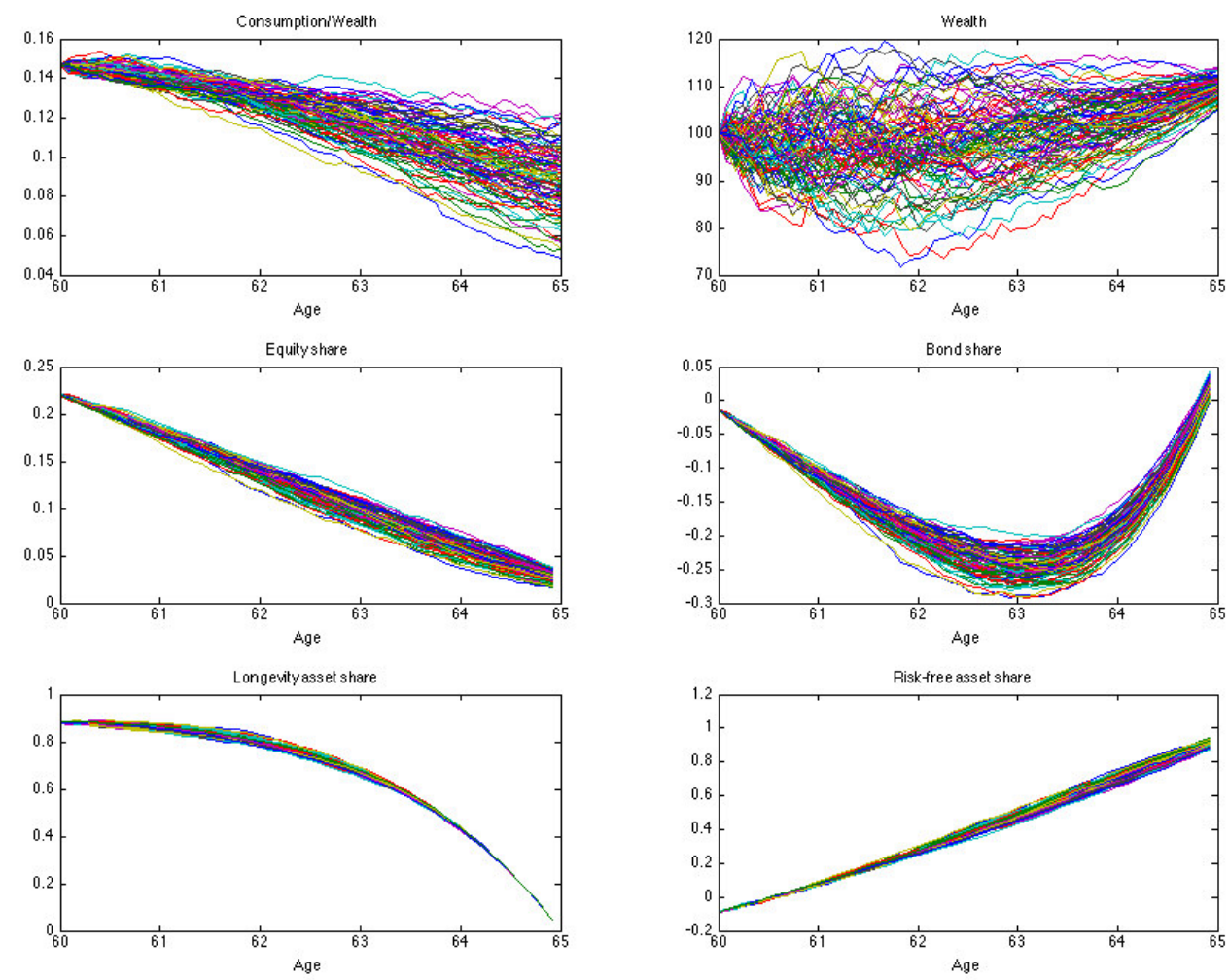
Figure 3: Sample path of optimal portfolio with the values gathered in Table 1, with $\phi_{\lambda}=0.1$.
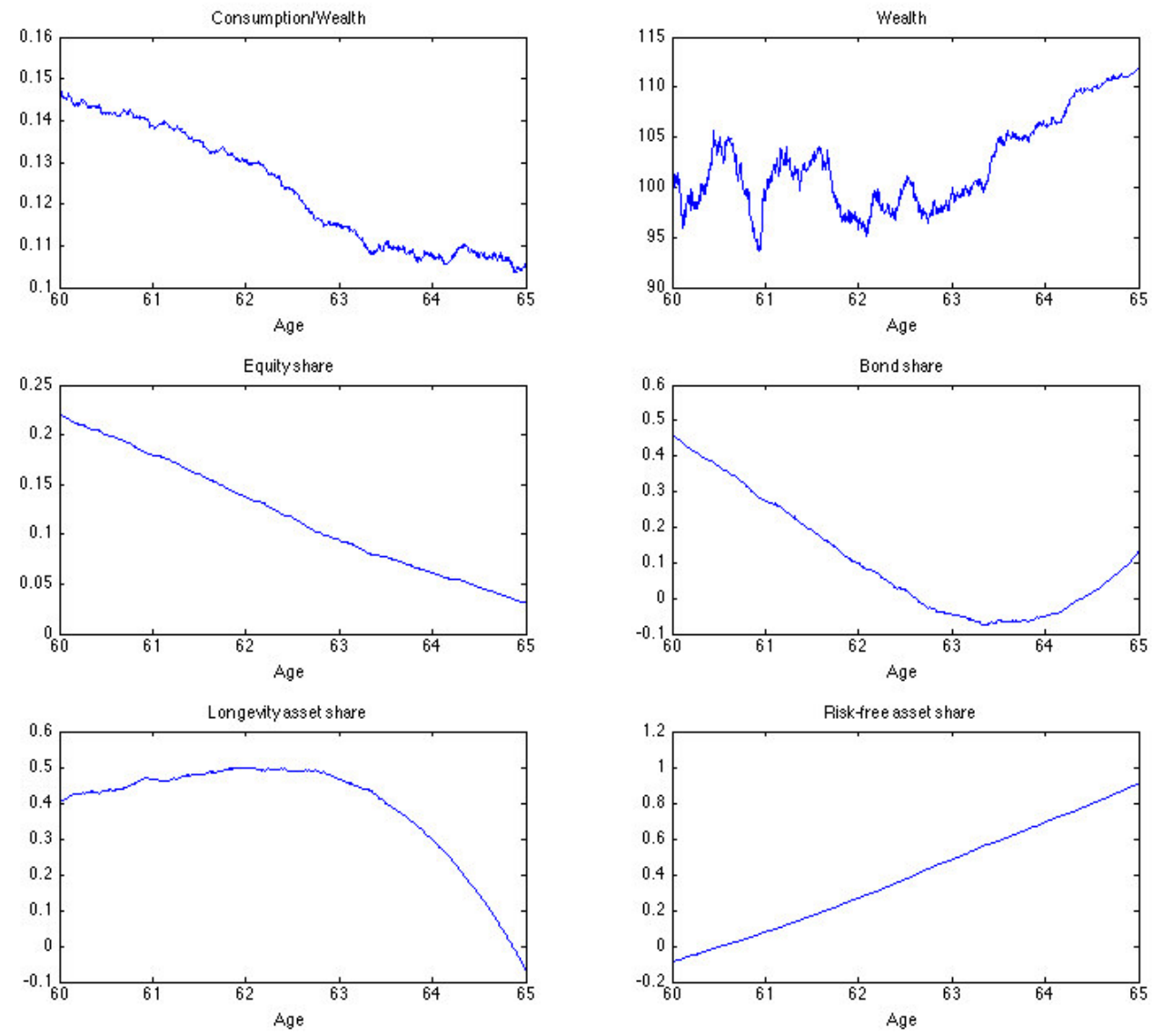
Figure 4: Sample path of optimal portfolio with the values gathered in Table 1, with $R_{m}=0$.
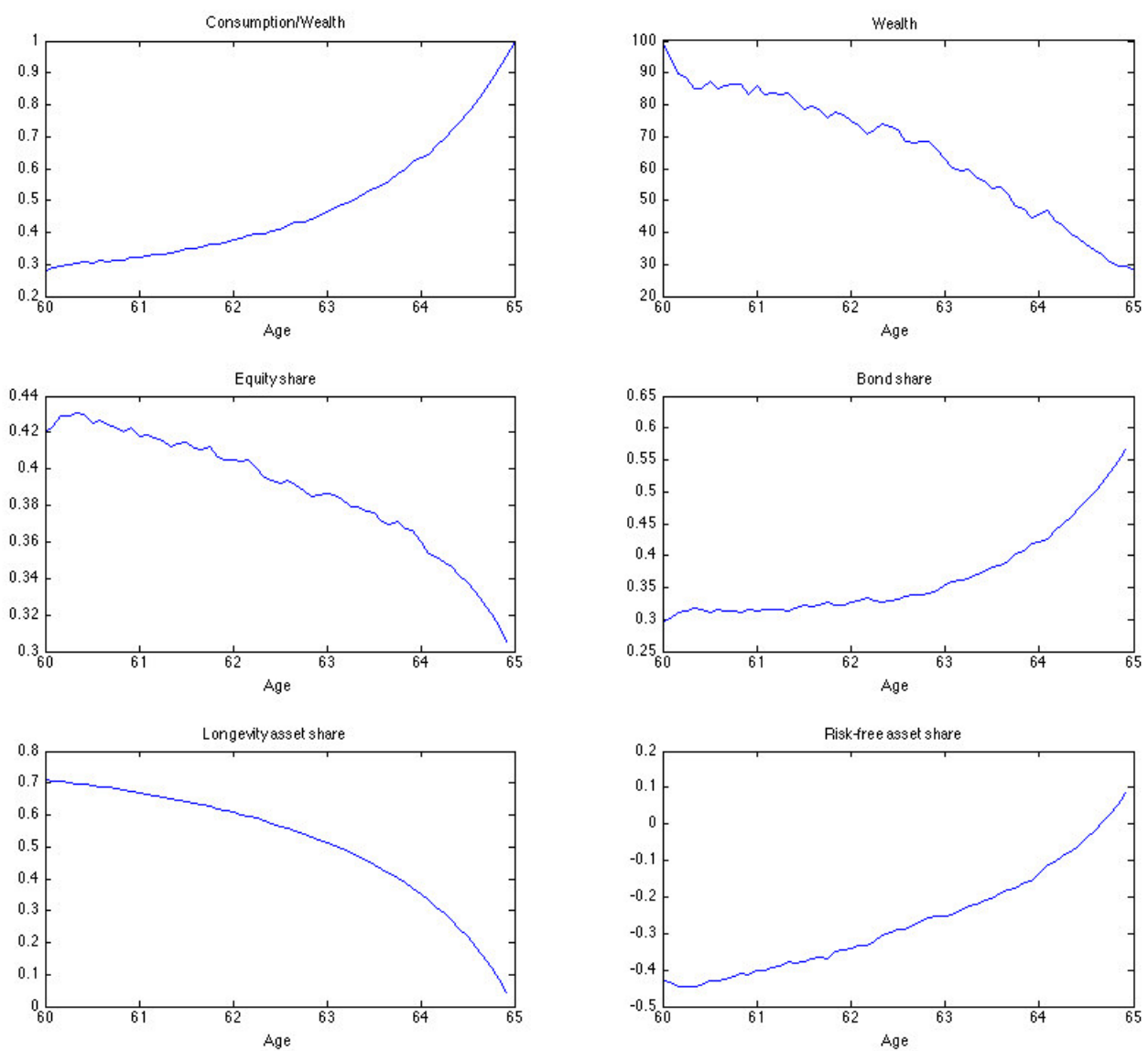


\section{Appendix A Proof of Proposition 1}

We solve problem (12) following the martingale approach. Its Lagrangian function under constraint (13) is:

$$
\begin{aligned}
\mathcal{L}= & \mathbb{E}_{t_{0}}\left[\int_{t_{0}}^{T} \frac{\left(c(s)-c_{m}\right)^{1-\delta}}{1-\delta} e^{-\int_{t_{0}}^{s} \rho(u)+\lambda(u) d u} d s+\frac{\left(R(T)-R_{m}\right)^{1-\delta}}{1-\delta} e^{-\int_{t_{0}}^{T} \rho(u)+\lambda(u) d u}\right] \\
& +\kappa\left(R\left(t_{0}\right)-\mathbb{E}_{t_{0}}\left[\int_{t_{0}}^{T}\left(c(s)-w(s)+\sigma_{L}(s)^{\prime} \xi(s)\right) m\left(t_{0}, s\right) e^{-\int_{t_{0}}^{s} r(u)+\lambda(u) d u} d s\right.\right. \\
& \left.\left.+R(T) m\left(t_{0}, T\right) e^{-\int_{t_{0}}^{T} r(u)+\lambda(u) d u}\right]\right),
\end{aligned}
$$

where the functional dependencies on $z$ have been omitted for the sake of simplicity, $\kappa$ is the (constant) Lagrangian multiplier, and all the expected values have been written under the historical probability. The first order condition on consumption

$$
\frac{\partial \mathcal{L}}{\partial c(s)}=\mathbb{E}_{t_{0}}\left[\int_{t_{0}}^{T}\left(\left(c(s)-c_{0}\right)^{-\delta} e^{-\int_{t_{0}}^{s} \rho(u)+\lambda(u) d u}-\kappa m\left(t_{0}, s\right) e^{-\int_{t_{0}}^{s} r(u)+\lambda(u) d u}\right) d s\right]=0,
$$

must hold for any state of the world and, accordingly, the optimal consumption at any time $s$ is

$$
c^{*}(s)=c_{m}+\left(\kappa m\left(t_{0}, s\right) e^{-\int_{t_{0}}^{s} r(u) d u} e^{\int_{t_{0}}^{s} \rho(u) d u}\right)^{-\frac{1}{\delta}} .
$$

The same approach on final wealth gives the first order condition

$$
\frac{\partial \mathcal{L}}{\partial R(T)}=\mathbb{E}_{t_{0}}\left[\left(R(T)-R_{m}\right)^{-\delta} e^{-\int_{t_{0}}^{T} \rho(u)+\lambda(u) d u}-\kappa m\left(t_{0}, T\right) e^{-\int_{t_{0}}^{T} r(u)+\lambda(u) d u}\right]=0
$$


and the optimal final wealth

$$
R^{*}(T)=R_{m}+\left(\kappa m\left(t_{0}, T\right) e^{-\int_{t_{0}}^{T} r(u) d u} e^{\int_{t_{0}}^{T} \rho(u) d u}\right)^{-\frac{1}{\delta}}
$$

When the constraint is rewritten at time $t$ (instead of $t_{0}$ ) as follows

$$
\begin{aligned}
R(t) & =\mathbb{E}_{t}\left[\int_{t}^{T}\left(c(s)-w(s)+\sigma_{L}(s)^{\prime} \xi(s)\right) m(t, s) e^{-\int_{t}^{s} r(u)+\lambda(u) d u} d s\right. \\
& \left.+R(T) m(t, T) e^{-\int_{t}^{T} r(u)+\lambda(u) d u}\right]
\end{aligned}
$$

and the optimal consumption and final wealth are both substituted in it, we obtain the following expression:

$$
R(t)=\left(\kappa m\left(t_{0}, t\right) \frac{e^{-\int_{t_{0}}^{t} r(u) d u}}{e^{-\int_{t_{0}}^{t} \rho(u) d u}}\right)^{-\frac{1}{\delta}} F(t, z)+H(t, z)
$$

where

$$
H(t, z)=\mathbb{E}_{t}^{\mathbb{Q}}\left[\int_{t}^{T}\left(c_{m}-w(s)+\sigma_{L}(s)^{\prime} \xi(s)\right) e^{-\int_{t}^{s} r(u)+\lambda(u) d u} d s+R_{m} e^{-\int_{t}^{T} r(u)+\lambda(u) d u}\right]
$$

$F(t, z)=\mathbb{E}_{t}\left[\int_{t}^{T} m(t, s)^{1-\frac{1}{\delta}} e^{-\int_{t}^{s}\left(\frac{\delta-1}{\delta} r(u)+\frac{1}{\delta} \rho(u)+\lambda(u)\right) d u} d s+m(t, T)^{1-\frac{1}{\delta}} e^{-\int_{t}^{T}\left(\frac{\delta-1}{\delta} r(u)+\frac{1}{\delta} \rho(u)+\lambda(u)\right) d u}\right]$.

While $m(t, s)^{1-\frac{1}{\delta}}$ is not a martingale, $m(t, s)^{1-\frac{1}{\delta}} e^{\frac{1}{2} \frac{1}{\delta} \frac{\delta-1}{\delta} \int_{t_{0}}^{t} \xi^{\prime} \xi d s}$ is:

$$
\left(m(t, s)^{1-\frac{1}{\delta}} e^{\frac{1}{2} \frac{1}{\delta} \frac{\delta-1}{\delta} \int_{t_{0}}^{t} \xi^{\prime} \xi d s}\right)^{-1} d\left(m(t, s)^{1-\frac{1}{\delta}} e^{\frac{1}{2} \frac{1}{\delta} \frac{\delta-1}{\delta} \int_{t_{0}}^{t} \xi^{\prime} \xi d s}\right)=-\frac{\delta-1}{\delta} \xi(s) d W(s) .
$$


Accordingly, we define the new probability

$$
d W(t)^{\mathbb{Q} \delta}=\frac{\delta-1}{\delta} \xi(t) d t+d W(t)
$$

and write

$$
F(t)=\mathbb{E}_{t}^{\mathbb{Q}_{\delta}}\left[\begin{array}{c}
\int_{t}^{T} e^{-\int_{t}^{s}\left(\frac{\delta-1}{\delta} r(u, z)+\frac{1}{\delta} \rho(u, z)+\lambda(u, z)+\frac{1}{2} \frac{1}{\delta} \frac{\delta-1}{\delta} \xi(u, z)^{\prime} \xi(u, z)\right) d u} d s \\
+e^{-\int_{t}^{T}\left(\frac{\delta-1}{\delta} r(u, z)+\frac{1}{\delta} \rho(u, z)+\lambda(u, z)+\frac{1}{2} \frac{1}{\delta} \frac{\delta-1}{\delta} \xi(u, z)^{\prime} \xi(u, z)\right) d u}
\end{array}\right]
$$

The differential of (A.7), through Ito's lemma, is (the drift term is neglected since it is immaterial to replication):

$$
\begin{aligned}
d R(t)= & (\ldots) d t+\frac{1}{\delta}\left(\kappa m\left(t_{0}, t\right) e^{-\int_{t_{0}}^{t} r(u) d u} e^{\int_{t_{0}}^{t} \rho(u) d u}\right)^{-\frac{1}{\delta}} F(t, z) \xi(t, z)^{\prime} d W(t) \\
& +\left(\kappa m\left(t_{0}, t\right) e^{-\int_{t_{0}}^{t} r(u) d u} e^{\int_{t_{0}}^{t} \rho(u) d u}\right)^{-\frac{1}{\delta}} F_{z}(t, z)^{\prime} \Omega(t, z)^{\prime} d W(t) \\
& +H_{z}(t, z)^{\prime} \Omega(t, z)^{\prime} d W(t)
\end{aligned}
$$

where the subscripts on $F(t, z)$ and $H(t, z)$ indicate partial derivatives. Once the following relationship

$$
\frac{R(t)-H(t, z)}{F(t, z)}=\left(\kappa m\left(t_{0}, t\right) e^{-\int_{t_{0}}^{t} r(u, z) d u} e^{\int_{t_{0}}^{t} \rho(u, z) d u}\right)^{-\frac{1}{\delta}}
$$

is suitably taken into account, the differential equation becomes

$d R(t)=(\ldots) d t+\left(\frac{R(t)-H(t, z)}{\delta} \xi(t, z)^{\prime}+\frac{R(t)-H(t, z)}{F(t, z)} F_{z}(t, z)^{\prime} \Omega(t, z)^{\prime}+H_{z}(t, z)^{\prime} \Omega(t, z)^{\prime}\right) d W(t)$. 
When $\Sigma(t, z) I_{S} \theta_{S}(t)+\sigma_{L}(t, z)$ is set equal to the diffusion term of (A.15), the optimal portfolio in Proposition 1 is found.

\section{Appendix B Proof of Proposition 2}

Let $y(t)$ solve the stochastic differential equation

$$
d y(t)=\alpha(\beta(t)-y(t)) d s+\sigma \sqrt{y(t)} d W(t) .
$$

If $\chi$ is constant, then the expected value $\mathbb{E}_{t}\left[e^{-\chi \int_{t}^{T} y(u) d u}\right]$ can be written as the exponential of an affine transformation of $y(t), e^{-D(t)-C(t) y(t)}$, whose differential is

$$
\frac{d\left(e^{-D(t)-C(t) y(t)}\right)}{e^{-D(t)-C(t) y(t)}}=(\underbrace{-\frac{\partial D(t)}{\partial t}-\frac{\partial C(t)}{\partial t} y(t)-C(t) \alpha(\beta(t)-y(t))+\frac{1}{2} C(t)^{2} \sigma^{2} y(t)}_{\chi y(t)}) d t-C(t) \sigma \sqrt{y(t)} d W(t)
$$

The drift differential equation can be split into two differential equations, one which depends on $y(t)$ and one which does not:

$$
\begin{gathered}
0=-\frac{\partial D(t)}{\partial t}-C(t) \alpha \beta(t) \\
0=-\frac{\partial C(t)}{\partial t} y(t)+C(t) \alpha y(t)+\frac{1}{2} C(t)^{2} \sigma^{2} y(t)-\chi y(t),
\end{gathered}
$$

whose boundary conditions are $D(T)=C(T)=0 . C(t)$ is obtained as the solution of a Riccati equation with constant coefficients, which is well known and given by (27). Once $C(t)$ has been computed, $D(t)$ is obtained from the PDE (B.3) and the corresponding boundary condition:

$$
D(t)=\alpha \int_{t}^{T} \beta(s) C(s) d s
$$




\section{Acknowledgements}

The Authors would like to thank conference participants to the 2nd PARTY 2015 Conference (Liverpool). Luca Regis gratefully acknowledges financial support from the CrisisLab project funded by the Italian government.

\section{References}

Barrieu, P., Bensusan, H., El Karoui, N., Hillairet, C., Loisel, S., Ravanelli, C., Salhi, Y., 2012. Understanding, modelling and managing longevity risk: key issues and main challenges. Scandinavian Actuarial Journal 3, 203-231.

Battocchio, P., Menoncin, F., Scaillet, O., 2007. Optimal asset allocation for pension funds under mortality risk during the accumulation and decumulation phases. Annals of Operations Research 152 (1), 141-165.

Bauer, D., Börger, M., Ruß, J., 2010. On the pricing of longevity-linked securities. Insurance: Mathematics and Economics 46 (1), 139-149.

Bernasconi, M., Levaggi, R., Menoncin, F., 2015. Tax evasion and uncertainty in a dynamic context. Economics Letters 126, 171-175.

Blake, D. Cairns, A., Dowd, K., MacMinn, R., 2006. Longevity bonds: financial engineering, valuation, and hedging. The Journal of Risk and Insurance 73 (4), 647-672.

Cocco, J., Gomes, F., 2012. Longevity risk, retirement savings, and financial innovation. Journal of Financial Economics 103 (3), 507-529. 
Dahl, M., 2004. Stochastic mortality in life insurance: market reserves and mortalitylinked insurance contracts. Insurance: Mathematics and Economics 35 (1), 113-136.

Delong, L., Gerrard, R., Haberman, S., 2008. Mean-variance optimization problems for an accumulation phase in a defined benefit plan. Insurance: Mathematics and Economics 42 (1), 107-118.

Denuit, M., Devolder, P., Goderniaux, A. C., 2007. Securitization of longevity risk: Pricing survivor bonds with wang transform in the lee-carter framework. Journal of Risk and Insurance 74 (1), 87-113.

Dybvig, P., Liu, H., 2010. Lifetime consumption and investment: Retirement and constrained borrowing. Journal of Economic Theory 145 (3), 885-907.

Farhi, E., Panageas, S., 2007. Saving and investing for early retirement: A theoretical analysis. Journal of Financial Economics 83 (1), 87-121.

Horneff, W., Maurer, R., Rogalla, R., 2010. Dynamic portfolio choice with deferred annuities. Journal of Banking \& Finance 34 (11), 2652-2664.

Huang, H., Milevsky, M., 2008. Portfolio choice and mortality-contingent claims: The general hara case. Journal of Banking \& Finance 32 (11), 2444-2452.

Huang, H., Milevsky, M. A., Salisbury, T. S., 2012. Optimal retirement consumption with a stochastic force of mortality. Insurance: Mathematics and Economics 51 (2), $282-291$.

Kwak, M., Lim, B., 2014. Optimal portfolio selection with life insurance under inflation risk. Journal of Banking \& Finance 46, 59-71. 
Lando, D., 1998. On cox processes and credit risky securities. Review of Derivatives Research 2 (2), 99-120.

Lee, R., Carter, L., 1992. Modeling and forecasting us mortality. Journal of the American statistical association 87 (419), 659-671.

Loeys, J., Panigirtzoglou, N., Ribeiro, R., 2007. Longevity: a market in the making. JPMorgan Global Market Strategy.

Luciano, E., Regis, L., 2014. Risk-return appraisal of longevity swaps. In: Pension and Longevity Risk Transfer for Institutional Investors. Vol. Fall 2014. Institutional Investor Journals, pp. 99-108.

Maurer, R., Mitchell, O., Rogalla, R., Kartashov, V., 2013. Lifecycle portfolio choice with systematic longevity risk and variable investment-linked deferred annuities. Journal of Risk and Insurance 80 (3), 649-676.

Menoncin, F., 2008. The role of longevity bonds in optimal portfolios. Insurance: Mathematics and Economics 42 (1), 343-358.

Menoncin, F., 2009. Death bonds with stochastic force of mortality. In: Vanmaele, M., Deelstra, G., De Schepper, A., Dhaene, J., Van Goethem, P. (Eds.), Actuarial and Financial Mathematics Conference - Interplay between finance and insurance.

Merton, R. C., 1971. Optimum consumption and portfolio rules in a continuous-time model. Journal of economic theory 3 (4), 373-413.

Milevsky, M., Promislow, D., 2001. Mortality derivatives and the option to annuitise. Insurance: Mathematics and Economics 29 (3), 299-318. 
Pirvu, T., Zhang, H., 2012. Optimal investment, consumption and life insurance under mean-reverting returns: The complete market solution. Insurance: Mathematics and Economics 51 (2), 303-309.

Yaari, M. E., 1965. Uncertain lifetime, life insurance, and the theory of the consumer. The Review of Economic Studies, 137-150. 


\section{Institution Markets \\ IMT}

INSTITUTE FOR ADVANCED STUDIES LUCCA

2015 ( ) IMT Institute for Advanced Studies, Lucca Piazza San ponziano 6, 5100 Lucca, Italy. www.imtlucca.it 